\title{
Multicolor infrared detection using two stacks of superlattice structures in a back-to-back configuration
}

\author{
M. C. Hsu and C. H. Kuan \\ Department of Electrical Engineering, National Taiwan University, Taipei, Taiwan, Republic of China \\ S. Y. Wang \\ Department of Electronics Engineering, National Chiao Tung University, Hsinchu, Taiwan, \\ Republic of China
}

(Received 25 February 2000; accepted for publication 17 July 2000)

\begin{abstract}
Voltage-controlled multicolor detection with a two-stack GaAs/AlGaAs superlattice structure is reported. Each stack contains a superlattice and current blocking layers. The responsivity of each stack has a voltage-tunable spectral range and can be turned on or off by controlling the bias polarity. In a back-to-back configuration of the two stacks, a variable detection range with peak wavelength at 5.5, 6.8, 8.5, and $10.8 \mu \mathrm{m}$ is achieved by changing the applied voltage at $45 \mathrm{~K}$. (C) 2000 American Institute of Physics. [S0003-6951(00)05037-3]
\end{abstract}

Using multicolor detection to enhance the performance of infrared detection, such as for object discrimination and temperature sampling, ${ }^{1,2}$ has attracted much investigation. Due to the flexibility in tailoring the detection wavelength and the spectral line shape, several designs utilizing intersubband transitions have been proposed. ${ }^{3-8}$ In particular, using stacks of multiple quantum wells (MQWs) to achieve multicolor detection is one the most common approaches. ${ }^{6-8}$ In this approach, although the number of detection bands increases with increasing number of stacks, the number of contacts required to access all stacks will also increase. This complicates the fabrication process. To avoid the additional contacts, efforts have been made to develop the voltagecontrolled multicolor detectors to select the desired band via the control of the bias voltage. ${ }^{6-8}$ However, the selectivity still remains to be improved and the bias requirement is usually too high $(>8 \mathrm{~V})^{7}$ in these detectors. Photodetectors utilizing the interminiband transitions in superlattices (SLs) have been demonstrated to operate at zero or low bias voltage. ${ }^{9-13}$ To reduce the large ground-stated tunneling current, most superlattice infrared photodetectors (SLIPs) were designed with a current blocking layer. In our previous study, ${ }^{13}$ we found that the current blocking barrier in such a SLIP may also serve as an energy filter for the photoexcited carriers and result in a voltage-tunable spectral responsivity. In addition, the responsivity was also found to be polarity selective. It can only be turned on in one bias polarity in which the photoexcited carriers are driven from the SL to pass the blocking barrier. The tunable, polarity selective, and low-bias features of such SLIPs thus can be used to improve the selectivity and bias requirement of voltage-controlled multicolor detectors. In this letter, we present the multicolor detection with two such SLIPs in the back-to-back configuration as shown in Fig. 1. The characterization of each individual SLIP and the integrated two-stack SLIP will be given. By changing the applied voltage on the two-stack SLIP, a variable detection range with peak wavelength at 5.5, 6.8, 8.5, and $10.8 \mu \mathrm{m}$, respectively, is achieved.

The sample was grown by molecular beam epitaxy on a semi-insulating GaAs substrate. The bottom (long- wavelength) SLIP consists of a $1500 \AA \mathrm{Al}_{0.20} \mathrm{Ga}_{0.80} \mathrm{As}$ blocking layer and 10 periods of $68 \AA$ GaAs well $(n=1$ $\times 10^{18} \mathrm{~cm}^{-3}$ ) and $35 \AA$ undoped $\mathrm{Al}_{0.27} \mathrm{Ga}_{0.73} \mathrm{As}$ barrier. The top (short-wavelength) SLIP consists of a $1500 \AA$ $\mathrm{Al}_{0.34} \mathrm{Ga}_{0.66} \mathrm{As}$ blocking layer and 10 periods of $45 \AA$ undoped $\mathrm{Al}_{0.34} \mathrm{Ga}_{0.66} \mathrm{As}$ barrier and $40 \AA \mathrm{AaAs}$ well $(n=1$ $\left.\times 10^{18} \mathrm{~cm}^{-3}\right)$. The two SLIPs are sandwiched between two $n^{+}$-GaAs contact layers $\left(n=1 \times 10^{18} \mathrm{~cm}^{-3}\right)$ and separated by a $4000 \AA n^{+}$-GaAs layer $\left(n=1 \times 10^{18} \mathrm{~cm}^{-3}\right)$. The sample was processed into a two-step square mesa with three terminal contacts, as shown in the inset of Fig. 2, to allow the characterization of each individual SLIP. The dimension of top square mesa is $150 \times 150 \mu \mathrm{m}^{2}$ and that of bottom mesa is $200 \times 400 \mu \mathrm{m}^{2}$.

The photoresponse and absorption spectra were measured using a Fourier transform infrared spectrometer (FTIR). For the photoresponse measurements, the detector under test was back illuminated through a polished $45^{\circ}$ facet on the substrate. The photoresponse current was first amplified by a current preamplifier then fed into the FTIR. The absolute responsivity was calibrated by using an unpolarized blackbody source. To keep both component SLIPs operating under the background limited performance, the detector was cooled down to $45 \mathrm{~K}$ during the photoresponse measurements. However, the absorption spectra were measured at room temperature. To obtain the absorption spectrum of each individual SLIP, first the absorption spectrum of the sample, with contributions from both SLIPs, was taken. The absorption of the bottom SLIP was then measured by removing the

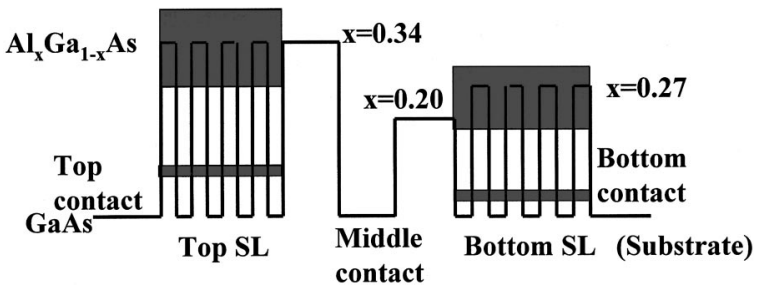

FIG. 1. Schematic energy band diagram of the two-stack SLIP. Each stack contains a SL and a current blocking layers. 

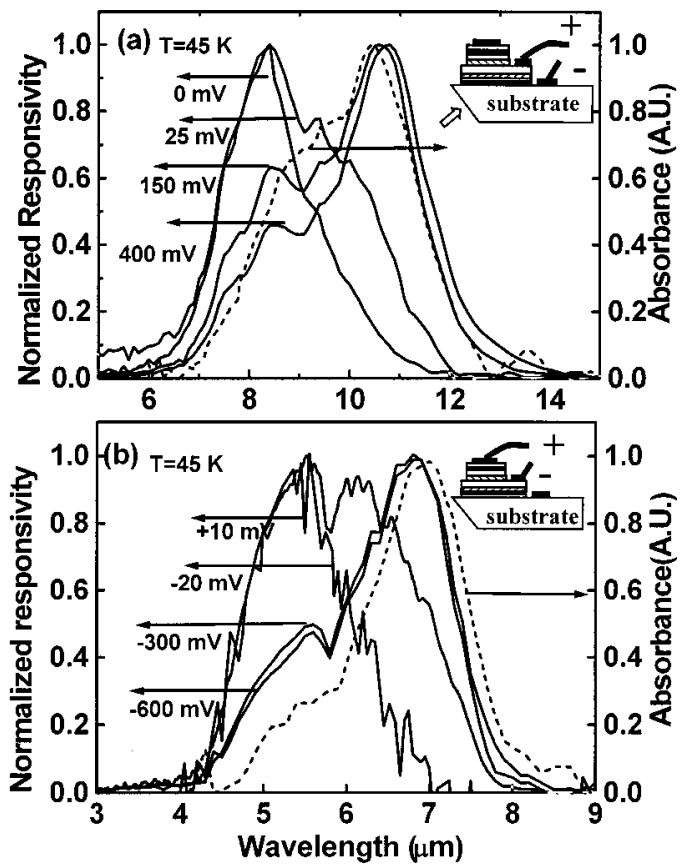

FIG. 2. The normalized response spectra of the bottom (a) and top (b) SLIPs. The dash curves are the associated absorption spectra measured at room temperature. The inset in each figure schematically shows the fabricated two-step mesa detector with the 45 deg facet on the substrate and the associated positive bias polarity.

top SLIP layer with wet chemical etching. Finally, the absorption of the top SLIP was obtained by subtracting the contribution of the bottom SLIP from the sample

The individual absorption spectra of the top and bottom SLIPs are shown in Figs. 2(a) and 2(b), respectively. In addition to the absorption spectra, Fig. 2 also shows the normalized spectral responsivities of two individual SLIPs at several bias voltages. The spectral responsivity of each SLIP, though covering a different spectral range, varies with the bias voltage in a similar way. At near zero bias, compared with the associated absorption spectrum, the spectral responsivity is at a shorter wavelength range. With the increasing of the bias magnitude, the cutoff wavelength of the spectral responsivity extends to a longer wavelength. At even higher biases, for example larger than $150 \mathrm{mV}$ for the bottom SLIP, the line shape of the spectral responsivity becomes insensitive to the bias voltage with a peak wavelength and a spectral range closed to those of the associated absorption spectrum. The agreement between the absorption spectrum and the responsivity spectra at high biases indicates that the voltagetuning behavior should be dominated by the transport process of the photoexcited carriers rather than by the absorption. Assuming the transport process is mainly limited by the blocking barrier, the voltage-tuning behavior can be explained in the following way: At near zero bias voltage, only those photoelectrons with their energies higher than that of the barrier can contribute to the photoresponse. When we increase the bias voltage, the photoelectrons with the energy slightly lower than that of the barrier can then tunnel through the triangular tip of the barrier. Therefore, it results in a longer cutoff wavelength. With further increasing of the bias voltage, the tunneling probability will increase and the barrier finally becomes nearly transparent for all photoexcited electrons. The resulting photoresponse spectrum thus exhib-

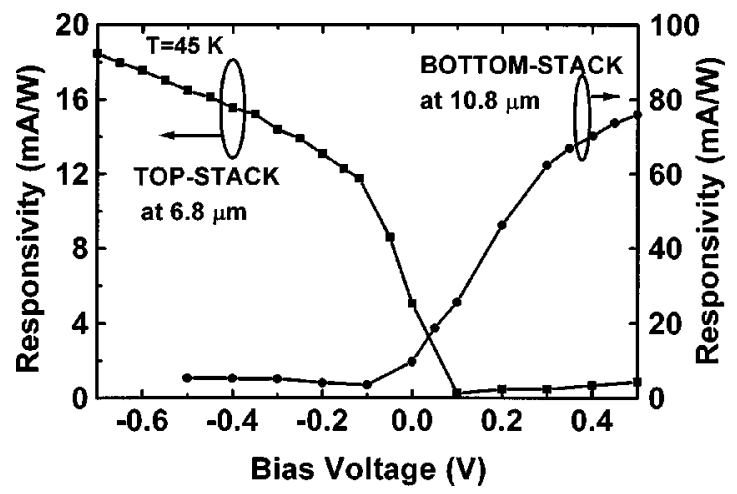

FIG. 3. The voltage dependence of the responsivity of top and bottom SLIPs.

its a spectral range and a peak position closed to those of the absorption.

The responsivity of each individual SLIP versus the bias voltage is shown in Fig. 3. We can see that each SLIP is turned on only when the applied voltage drives the photoelectrons generated from the SL layer to pass the blocking layer. When the photoelectrons are driven in an opposite direction, the responsivity is nearly negligible. The polarityselective feature in such an asymmetrical structure may be attributed to the fact that when those photoexcited carriers are driven in the turned-off direction, the reinjection carriers from the collector contact to the SL layer are prevented by the blocking barrier.

The spectral responsivity of the two stacks in the backto-back configuration as a two-terminal device is shown in Fig. 4. The resulting spectra are similar to those in Fig. 2. Under negative biases the photoresponse is mainly contributed from top SLIP, while under positive biases the photoresponse is from the bottom SLIP. The peak wavelengths are $5.5,6.8,8.5$, and $10.8 \mu \mathrm{m}$ at $-0.02,-1,0.75$, and $1.2 \mathrm{~V}$, respectively. These results show the excellent voltagecontrolled capability of our device utilizing the polarityselective and voltage-tunable responsivity in each component SLIP.

In order to see the relation between the magnitude of responsivity and bias voltage, the responsivities versus bias voltage for wavelengths of 6.8 and $10.8 \mu \mathrm{m}$ were measured, as shown in Fig. 5. The responsivity for $10.8 \mu \mathrm{m}$ reaches the maximum with $65 \mathrm{~mA} / \mathrm{W}$ at about $1.2 \mathrm{~V}$, and is turned off at negative biases. Similarly, the responsivity for $6.8 \mu \mathrm{m}$ reaches its maximum with $17 \mathrm{~mA} / \mathrm{W}$ at about $-1 \mathrm{~V}$ and is

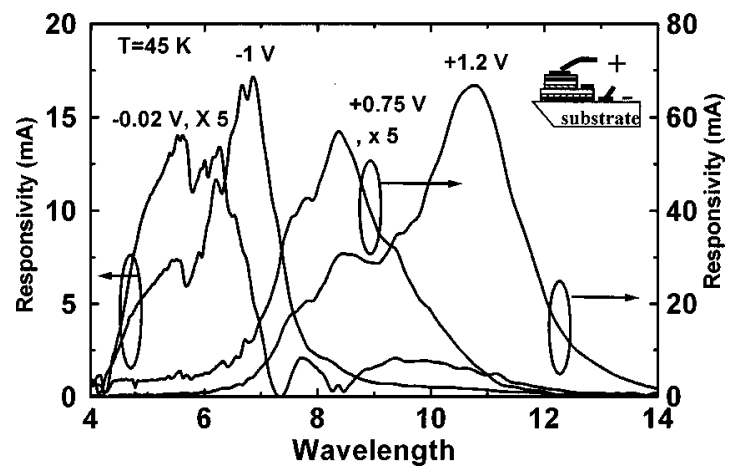

FIG. 4. The spectral responsivity at four biases applied between the top and bottom contacts. 


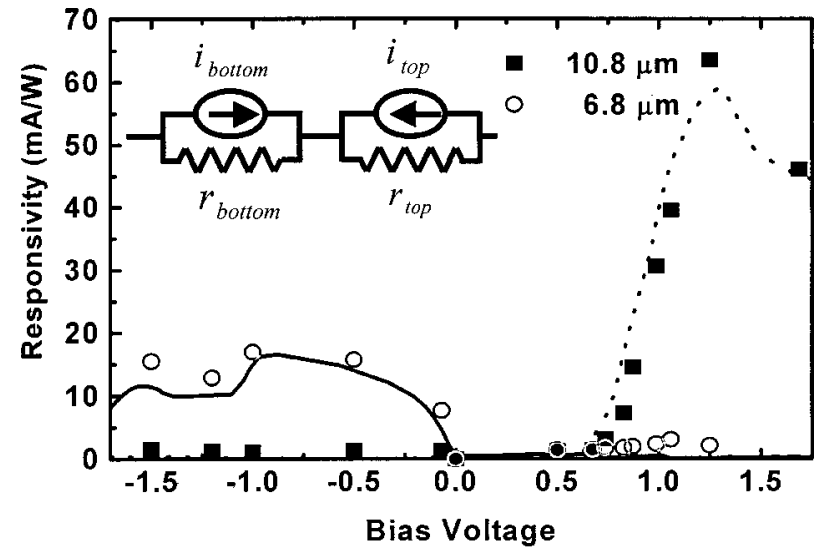

FIG. 5. Measured responsivities for $6.8(\bigcirc)$ and $10.8 \mu \mathrm{m}(\mathbf{a})$ vs applied voltage. The solid and dash curves represent the responsivities estimated from Eq. (1). The inset shows the small-signal equivalent circuit for the two-stack SLIP.

turned off at positive biases. The relation between the responsivity and the bias voltage can be further analyzed with the small signal equivalent-circuit model in Ref. 7. According to this model, the equivalent circuit of a two-stack SLIP involves a network of photocurrent sources and the dynamic differential resistances as shown in the inset of Fig. 5. The measured photoresponse current of our two-stack SLIP then is estimated by

$$
i_{p}=\frac{i_{p, \mathrm{bott}} r_{\mathrm{bott}}+i_{p, \mathrm{top}} r_{\mathrm{top}}}{r_{\mathrm{bott}}+r_{\mathrm{top}}},
$$

(Ref. 7), where $i_{p \text {, bott }}\left(i_{p \text {,top }}\right)$ and $r_{\text {bott }}\left(r_{\text {top }}\right)$ represent the photoresponse current and the dynamic resistance for the bottom(top) SLIP. The dash and solid curves in Fig. 5 represent the responsivities calculated by using Eq. (1). They agree well with the experimental data.

Since the equivalent circuit model works well in the analysis of the photoresponse current, we also use it to evaluate the noise current of the two-stack SLIP. Our previous study shows that the noise performance for a single-stack SLIP exhibits the full shot noise. ${ }^{13}$ We therefore estimate the noise current in the whole detector by

$$
i_{n}^{2}=2 e I_{\mathrm{dc}} \frac{r_{\text {bott }}^{2}+r_{\text {top }}^{2}}{\left(r_{\text {bott }}+r_{\text {top }}\right)^{2}} B,
$$

where $I_{\mathrm{dc}}$ is the bias current, $i_{n}$ the noise current, and $B$ the measurement bandwidth. With the estimated noise currents, the detectivities of the device are estimated as $1 \times 10^{9}, 4$ $\times 10^{9}, 1.5 \times 10^{9}$, and $5 \times 10^{9} \mathrm{~cm} \mathrm{~Hz}^{1 / 2} / \mathrm{W}$ at the bias voltages of $0.02,-1,0.75$, and $1.2 \mathrm{~V}$, respectively.

In summary, we have investigated the performance of a two-stack SLIP as a voltage-controlled multicolor detector. Using the polarity selective responsivity and the voltagetunable cutoff wavelength of each individual SLIP, we successfully select four peak wavelengths at 10.8, 8.5, 6.8, and $5.5 \mu \mathrm{m}$ from the detector with the bias voltage. Following our proposed design and incorporating InGaAs composition in the well region for the short-wavelength stack, it should be possible to fabricate a similar detector with a variable detection range covering the $3-5$ and 8-12 atmospheric windows simultaneously. Compared with the traditional voltagecontrolled designs with the multiple quantum well structures, the detector provides an attractive alternative which utilize the switching operation between the two active stacks with a moderated bias voltage $(<1.5 \mathrm{~V})$ for voltage-controlled multicolor detection.

This work is supported by National Council of Republic of China under the Contract No. NSC89-2215-E-002-019. The authors would like to thank Professor C. P. Lee of ChaoTung University for his assistance in this work.

${ }^{1}$ R. M. Spitzberg, Opt. Eng. 33, 2419 (1994).

${ }^{2}$ C. J. Chen, K. K. Choi, W. H. Chang, and D. C. Tsui, Appl. Phys. Lett. 72, 7 (1998).

${ }^{3}$ B. F. Levine, C. G. Bethea, B. O. Shen, and R. J. Malik, Appl. Phys. Lett. 57, 383 (1990).

${ }^{4}$ Jung-Chi Chiang, and Sheng S. Li; M. Z. Tidrow; P. Ho, M. Tsai, and C. P. Lee, Appl. Phys. Lett. 69, 2412 (1996).

${ }^{5}$ M. Martinet, F. Luc, E. Rosencher, Ph. Bois, and S. Delaitre, Appl. Phys. Lett. 60, 895 (1992).

${ }^{6}$ I. Grave, A. Shakouri, N. Kuze, and A. Yariv, Appl. Phys. Lett. 60, 2362 (1992).

${ }^{7}$ H. C. Liu, J. Li, J. R. Thompson, Z. R. Wasilewski, M. Buchanan, and J. G. Simmins, IEEE Electron Device Lett. 14, 566 (1993).

${ }^{8}$ K. L. Tsai, C. P. Lee, J. S. Tsang, and H. R. Chen, Electron. Lett. 30, 1352 (1994).

9 Kastalsky, T. Duffield, S. J. Allen, and J. Harbison, Appl. Phys. Lett. 52, 1320 (1988)

${ }^{10}$ Byungsung O, J-W. Choe, M. H. Francombe, K. M. S. V. Bandara, D. D. Coon, Y. F. Lin, and W. J. Takei, Appl. Phys. Lett. 57, 503 (1990).

${ }^{11}$ C. Shin Wu, Chen P. Wen, R. N. Sato, M. Hu, C. W. Tu, J. Zhang, L. D. Flesner, L. Pham, and P. S. Nayer, IEEE Trans. Electron Devices 39, 234 (1992).

${ }^{12}$ K. M. S. Bandara, J-W. Choe, M. J. Francombe, A. G. U. Perera, and Y. F. Lin, Appl. Phys. Lett. 60, 3022 (1992).

${ }^{13}$ M. C. Hsu, Y. F. Hsu, and C. H. Kuan, IEEE Trans. Electron Devices 47, 944 (2000). 